\title{
Inhibition of histone deacetylases in cancer therapy: lessons from leukaemia
}

\author{
Elena Ceccacci ${ }^{1,2}$ and Saverio Minucci ${ }^{\star 1,2}$ \\ ${ }^{1}$ Department of Experimental Oncology and Drug Development Program, European Institute of Oncology, Via Adamello 16, \\ 20139 Milan, Italy and 2 Department of Biosciences, University of Milan, Via Celoria 26, 20133 Milan, Italy
}

Histone deacetylases (HDACs) are a key component of the epigenetic machinery regulating gene expression, and behave as oncogenes in several cancer types, spurring the development of HDAC inhibitors (HDACi) as anticancer drugs. This review discusses new results regarding the role of HDACs in cancer and the effect of HDACi on tumour cells, focusing on haematological malignancies, particularly acute myeloid leukaemia. Histone deacetylases may have opposite roles at different stages of tumour progression and in different tumour cell sub-populations (cancer stem cells), highlighting the importance of investigating these aspects for further improving the clinical use of HDACi in treating cancer.

Epigenetic mechanisms have a key role in the control of biological processes, and chromatin alterations may lead to the onset and progression of many diseases, first of all cancer.

Unlike genetic alterations, epigenetic alterations are generally reversible. For this reason, drugs acting against epigenetic targets (epidrugs) have been developed and some of them have been approved for selected cancer indications, thus validating the concept of epigenetic therapy.

Histones present a great number of modifications, including acetylation, methylation, phosphorylation, ubiquitination and many others, more recently identified. This pattern is sometimes referred as 'histone code', but the parallelism with a code represents an oversimplification, as similar combinations of histone marks may result in different functional outcomes depending on the context, and different combinations may lead to a similar functional result.

Among histone modifications, lysine acetylation depends on the antagonistic activity of two enzyme classes: histone acetylases (HATs) and histone deacetylases (HDACs, subject of this review).

The human HDAC family comprises 18 proteins that can be grouped into four classes on the basis of sequence homology with yeast proteins:

- Class I (HDAC 1-2-3-8) are homologous to the yeast Rpd3, localise in the nucleus and contain a single deacetylase domain at the $\mathrm{N}$ terminus.

- Class II HDACs can be further divided into two classes: IIa (HDAC 4-5-7-9, localised in the nucleus and cytoplasm) and IIb
(HDAC 6 and 10); HDAC6 is mainly located in the cytoplasm and contains two catalytic domains, whereas HDAC10 contains a functional $\mathrm{N}$-terminal domain and a C-terminal incomplete domain.

- Class III HDACs are also termed sirtuins (SIRT1-SIRT7), which are homologs of yeast Sirt2 and differ structurally from the other classes, requiring $\mathrm{NAD}+$ as a cofactor.

- Class IV contains a single HDAC (HDAC11) with a catalytic domain shared with classes I/II HDACs.

Here, we will focus on Classes I/II and IV HDACs. These HDACs mediate their function as part of large macromolecular complexes in association with other factors: HDAC1 and HDAC2 are found in the mSin3A, NURD and Co-REST complexes, and HDAC3 is found associated with N-CoR and SMRT, whereas several proteins involved in the ubiquitin pathway are found associated with HDAC6 (Minucci and Pelicci, 2006).

\section{HDACS AND THE CONTROL OF HISTONE (AND NON-} HISTONE) ACETYLATION

The addition of acetyl groups to lysine residues in the histone tails by HATs is responsible for a relaxed and accessible chromatin structure, and is associated with transcriptional activation; conversely, HDACs remove acetyl groups and lead to a more closed chromatin structure, generally associated with transcriptional repression.

*Correspondence: Professor S Minucci; E-mail: Saverio.minucci@ieo.eu

Received 24 July 2015; Received 7 January 2016; accepted 12 January 2016; published online 23 February 2016 
Genome-wide studies of HDACs by chromatin immunoprecipitation followed by next-generation sequencing (Chip-Seq) revealed, however, a strong association between HDACs and active genes, and suggested a role for HDACs also in active transcription: when associated with active genes, HDACs act to remove acetyl groups added by HATs during transcriptional initiation and elongation, providing a reset of the chromatin structure that is required for a second round of transcription. Indeed, excessive histone acetylation at transcribed regions could 'destabilise' chromatin, thus leading to an increase of transcription at erroneous starting sites (Wang et al, 2009). In Drosophila, the UpSET complex-that includes HDACs-is required to reduce spreading of histone acetylation from active promoter regions and therefore limiting transcriptional noise (Rincon-Arano et al, 2012). The canonical relationship between HDACs, low levels of histone acetylation and transcriptional repression is therefore not always valid.

Histone acetylases and HDACs are responsible for the reversible acetylation not only of histones but also of a large number of additional substrates such as transcription factors, DNA repair enzymes and nuclear and cytoplasmic proteins. Not all protein acetylation, however, necessarily derives from the enzymatic action of HATs/HDACs: protein acetylation in mitochondria may be a chemical event facilitated by the alkaline $\mathrm{pH}$ and high concentrations of reactive acetyl-CoAs present in the mitochondrial matrix (Wagner and Payne, 2013).

Acetylation of non-histone proteins and regulation of their function adds another layer of complexity to the action of HATs/ HDACs: importantly, this also precludes from considering HDACs as purely 'epigenetic factors' (Minucci and Pelicci, 2006).

Proteomic studies have very recently led to the discovery of a large number of novel histone post-translational modifications (PTMs) that show additional acyl moieties beside acetylation (Kebede et al, 2015); among those PTMs, propionylation, butyrylation, crotonylation, succinylation, malonylation, glutarylation and lysine 2-hydroxyisobutyrylation share the use of shortchain acyl-coAs derived from energy metabolism as cosubstrate(s). These histone PTMs contribute to transcriptional regulation by promoting DNA unwrapping and nucleosome disassembly, reducing nucleosome stability and influencing the action of chromatin-associated factors: intriguingly, HDACs are able to remove at least a subset of these newly discovered acyl-histone marks (Kebede et al, 2015).

Currently, it remains unclear whether concentration of coAs (depending on the metabolic status of the cell) could be a critical factor determining the type of histone acylation, and if HDACs could have a role in transmitting metabolic signals by modulating the chromatin structure (a function already known for class III HDACs, regulated by $\mathrm{NAD}+/ \mathrm{NADH}$ ratio) and which impact metabolism can have on histone modifications. Importantly, tumour cells show constant alterations in metabolism that may lead, therefore, to alterations in HDAC function, as recent studies begin to suggest (Chiaradonna et al, 2015).

HDACS IN CANCER AND A (WEAK) RATIONALE FOR THE DEVELOPMENT OF HDAC INHIBITORS

Given their pleiotropic roles and their involvement in essentially all cell functions, HDACs may not be considered at a first glance as attractive targets for therapy, owing to the likely interference of HDAC inhibitors with several processes occurring in normal cells and therefore high risk of side effects. Indeed, the first HDAC inhibitors were initially characterised for their antitumour activity in vitro before the discovery that they were known to inhibit HDACs: their use in preclinical models (in vitro and in vivo) showed a significant therapeutic window, with reduced effects on normal cells.

The discovery of the potent antitumoral effects following HDAC inhibition led to the hypothesis that HDACs themselves may act as oncogenes, and, in fact, distinct HDACs are found overexpressed in various solid tumours, in some cases showing a differential expression in tumour subtypes: as an example, HDAC1 is highly expressed in hormone receptor-positive breast cancer, whereas HDAC2 and HDAC3 are more expressed in breast cancers with a more aggressive phenotype including hormone receptor-negative cancers (Müller et al, 2013). It is difficult, however, to correlate the degree of expression of individual HDACs with particular functional consequences and biological phenotypes: loss of acetylation of lysine 16 and 20 of histone $\mathrm{H} 4$ has been observed in various cancer cell lines and primary tumours, and described as a hallmark of human cancer: this could be because of overexpression of the class III HDAC SIRT1, which is capable of deacetylating histone H4K16 (Fraga et al, 2005). Reduced acetylation because of enhanced expression of HDACs may lead to transcriptional repression of tumour-suppressive pathways, including cell cycle regulators and DNA repair pathways. Overexpression of HDACs has been linked (together with other histone modifications) to the epigenetic repression of the locus encoding for the tumour suppressor $C D K N 1 A$, and of DNA damage repair genes such as BRCA1 and ATR (Eot-Houllier et al, 2009), but the consequence of HDAC overexpression may also impact on non-histone substrates: HDAC1 and HDAC2 regulate acetylation of the oncosuppressor p53, thus inhibiting its function (Insinga et al, 2004).

Overexpression of HDACs has been proposed in several cases as a negative prognostic marker, independently of tumour type and disease progression; however, this is not always the case as high levels of HDAC6 predict better prognosis in ER-positive breast cancer (Saji et al, 2005), or in CTCL.

Although HAT encoding genes are found frequently mutated or amplified in cancer, with consequent loss or alteration of function, there are reports of only rare, inactivating mutations of HDACs in cancer, which may suggest oncosuppressive roles for HDACs (Ropero et al, 2006).

We performed a preliminary analysis of the mutational landscape of HDACs using available data sets, focusing on missense mutations in the coding sequence, without further investigating the mutational impact of each mutation (Figure 1 and Supplementary Table S1).

The frequency of missense mutations of HDACs varies greatly in different human cancers: although in some cases (melanoma, lung cancer) the high frequency of mutations (close to $30 \%$ considering all HDACs) is somewhat expected because of the general high mutation rate caused by exposure to carcinogens (sunlight, smoke), other cancer types show a rate of HDAC mutations that could only in part be explained by their general mutational trend. In general, class II HDACs show higher percentages of mutations, and the distribution of missense mutations in the two most mutated HDACs, HDAC4 and HDAC9, show that although most mutations are distributed along the entire coding sequence, and therefore they are unlikely to be functionally relevant, a few sites - located in the catalytic domain - mutated in a higher number of patients exist, suggesting a potential alteration of the enzyme function (Figure 1B).

Taken together, these observations (that need to be extended) hint that just looking at mutations and/or altered expression pattern will not provide frequently a conclusive answer, and that more in-depth mechanistical insights are required to understand the altered function of HDACs in cancer cells.

Indeed, HDAC inhibition affects markedly cancer cells, inducing (depending on drug, dosage and tumour cell type) cell cycle arrest, differentiation, induction of cell death, reduction of 
A

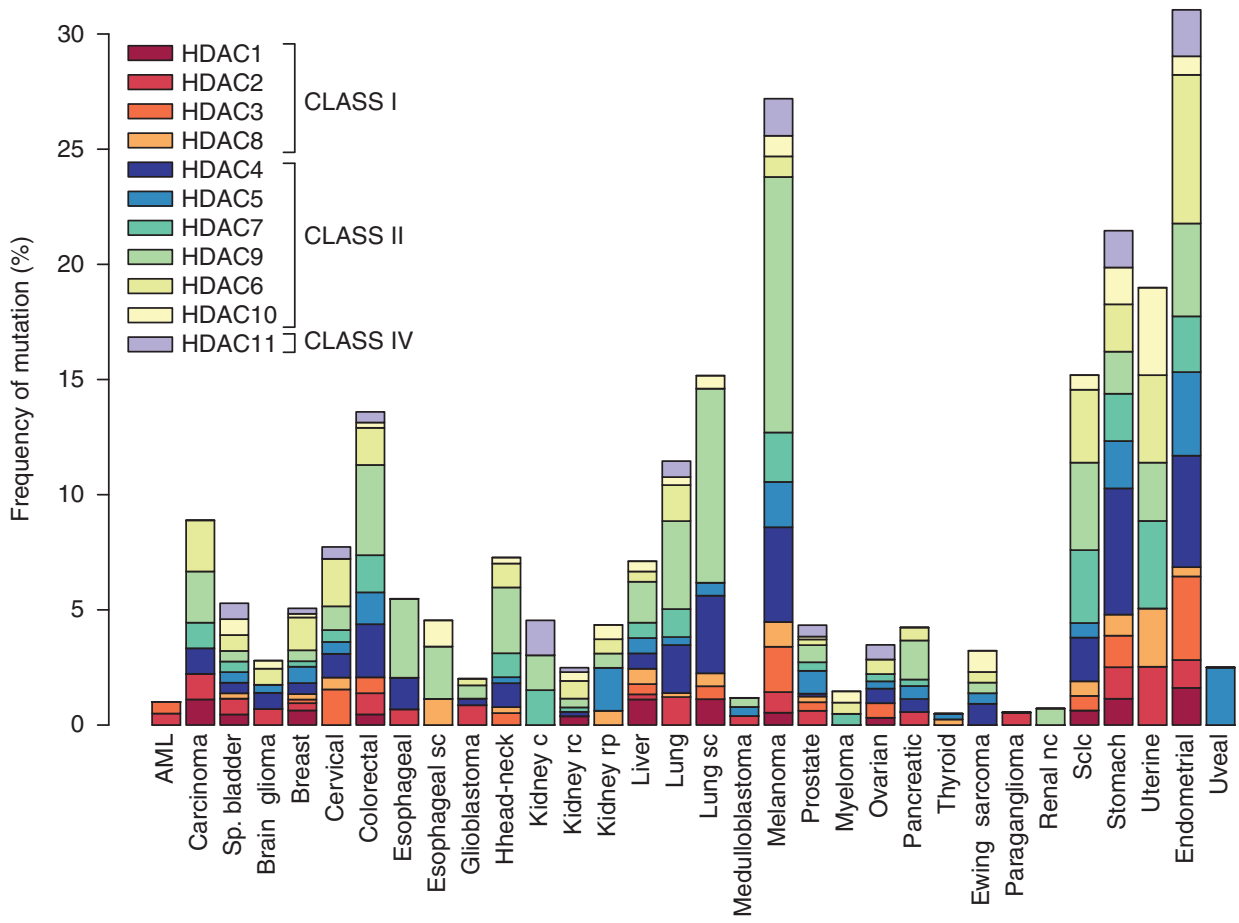

B
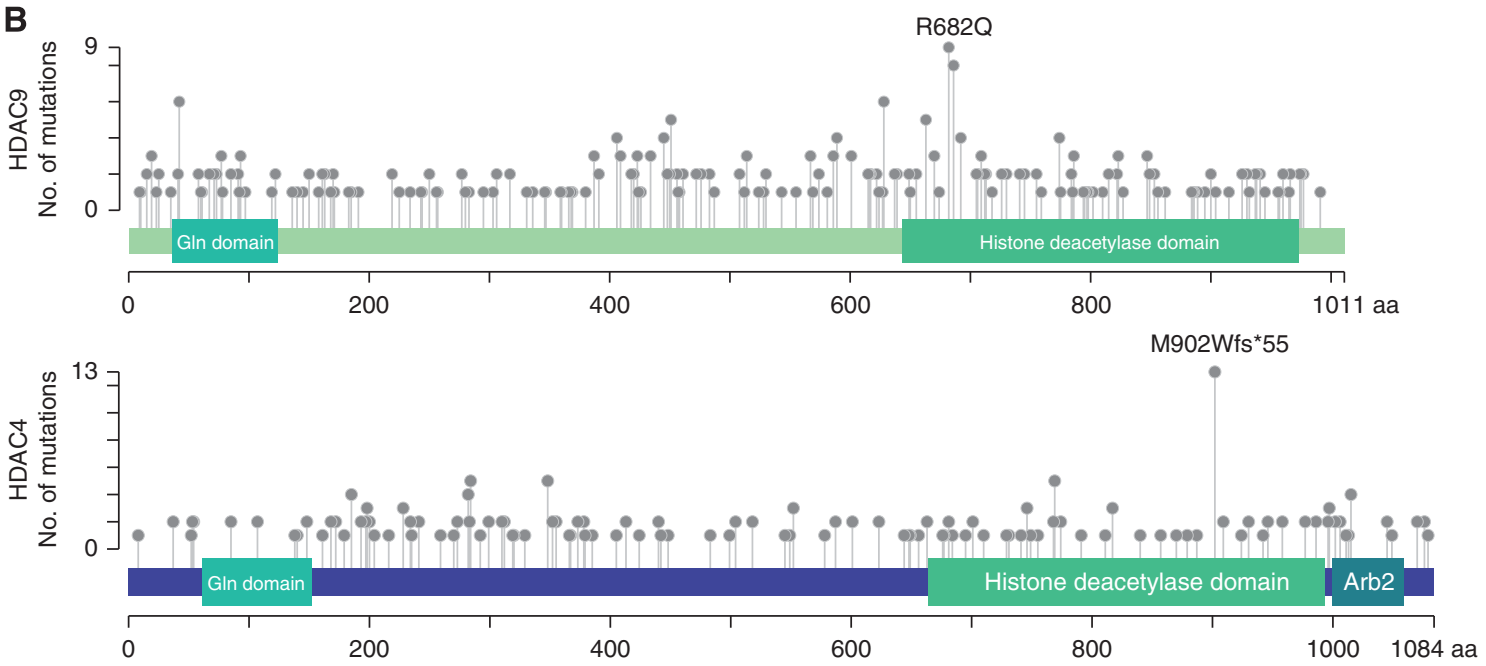

Figure 1. HDACs class I, II, IV mutations in human cancer. (A) Histone deacetylases class I, II and IV mutations across different human cancers. The histogram shows an overview of the frequency of mutations (missense) of each HDAC (classes I, II and IV) across different human cancers (analysis was performed on data downloaded from cBioPortal, see Supplementary Table S1 for a guide to the abbreviations, and the description for each cancer subtype of sample size). (B) Distribution of mutations across HDAC9 and HDAC4 coding sequences. Lolliplot graph of missense mutations found across all human cancers for HDAC9 (upper panel) and HDAC4 (lower panel). Note that though mutations are equally distributed along the entire coding sequence, in both cases the most frequent mutation is localised within the histone deacetylase catalytic domain. For HDAC4, in 13 patients the same mutation introduces a frameshift in the middle of the deacetylase domain, leading most likely to a functional inactivation of the domain. The two graphs were obtained by using the cBioPortal tool 'MutationMapper' (Cerami et al, 2012).

angiogenesis and modulation of the immune system. An 'epigenetic vulnerability' of tumour cells has been proposed, where - in contrast to normal cells that show redundancy in epigenetic regulatory mechanisms - HDACs may be essential in tumour cells for the maintenance of a set of key genes required for survival and growth (Dawson and Kouzarides, 2012).

A large number of HDAC inhibitors has been synthesised and tested in clinical trials, resulting in the approval of four inhibitors (Vorinostat, Romidepsin, Bellinostat, Panobinostat: a list of HDAC inhibitors, their classification and clinical status is provided in Table 1). This could be interpreted as a successful history of drug development, with validation of HDACs as important targets in cancer, but the situation is far more complex, and the clinical results do not reflect those expected from the preclinical work, both in terms of efficacy (observed only in selected cancer subtypes, mainly in haematology) and safety (several side effects were observed, among which the most common are fatigue, diarrhoea, bone marrow toxicity, thrombocytopenia; Subramanian et al, 2010). The reasons for this, at least in part, disappointing set of clinical results are not clear; one explanation could be the lack of selectivity of most of the HDAC inhibitors tested clinically and approved to date, acting as paninhibitors on all HDAC classes (not including sirtuins): the global inhibition of several non-redundant HDACs, with 
Table 1. HDAC inhibitors classified according to: (a) status of clinical advancement; (b) HDAC(s) targeted and (c) chemical class

\begin{tabular}{|c|c|c|c|}
\hline Compounds & Target & Class & Highest phase trial \\
\hline Panobinostat (LBH-589) & Pan-HDAC inhibitor & Hydroxamic acids & Approved in 2015 for multiple myeloma \\
\hline Belinostat (PXD101) & Pan-HDAC inhibitor & Hydroxamic acids & Approved in 2014 for PTCL \\
\hline Romidepsin (desipeptide-FK228) & Pan-HDAC inhibitor & Cyclic tetrapeptides & Approved in 2009 for CTCL \\
\hline SAHA(Vorinistat, Zolinza) & Pan-HDAC inhibitor & Hydroxamic acids & Approved in 2006 for CTCL \\
\hline Valproic acid & Pan-HDAC inhibitor & Short-chain fatty acids & Phase III \\
\hline Tacedinaline (C1994) & Subclass I-selective inhibitor (HDACs 1, 2 and 3) & Benzamides & Phase III \\
\hline Givinostat (ITF2357) & Pan-HDAC inhibitor & Hydroxamic acids & Phase II \\
\hline Resminostat (4SC201) & Pan-HDAC inhibitor & Hydroxamic acids & Phase II \\
\hline Abexinostat ( $\mathrm{PCl} 24781)$ & Pan-HDAC inhibitor & Hydroxamic acids & Phase II \\
\hline Rocilinostat (ACY1215) & Selective class II HDAC inhibitor & Hydroxamic acids & Phase II \\
\hline Quisinostat (JNJ-26481585) & Pan-HDAC inhibitor & Hydroxamic acids & Phase II \\
\hline Practinostat (SB939) & Inhibit class I, II and IV HDACs & Hydroxamic acids & Phase II \\
\hline Mocetinostat (MGCD0103) & Specific against class I and IV HDACs & Benzamides & Phase II \\
\hline Entinostat (MS275-SNDX-275) & Class I HDAC inhibitor & Benzamides & Phase II \\
\hline Sodium phenylbutyrate & Inhibit class I and II HDACs & Short-chain fatty acids & Phase II \\
\hline AR42 & Pan-HDAC inhibitor & Hydroxamic acids & Phase I \\
\hline 4SC202 & Selective class I HDAC inhibitor & Benzamides & Phase I \\
\hline Pyroxamide (NSC696085) & Inhibitor of affinity-purified HDAC1 & Hydroxamic acids & Phase I \\
\hline CHR-3996 & Selective class I HDAC inhibitor & Hydroxamic acids & Phase I \\
\hline CHR-2845 & & Hydroxamic acids & Phase I \\
\hline
\end{tabular}

partially overlapping but with clearly distinct and sometimes contrasting functions, may result in a difficult to predict phenotype, and subtle differences among species may explain the different results observed in murine models.

Indeed, knockout studies in mice on all members of class I HDACs demonstrated the unique roles of each HDAC in the control of specific gene expression programmes: HDAC1-null mice die at day E10.5 and display severe proliferation defects and general growth retardation; HDAC2-null mice die $24 \mathrm{~h}$ after birth for cardiac malformations; HDAC3-null mice die before E9.5 for defects in gastrulation probably because of defective DNA repair (Haberland et al, 2009; Yang and Seto, 2008).

The hypothesis that inhibition of specific HDACs may have a better therapeutic outcome will be put to test once we will evaluate more selective HDAC inhibitors in clinical trials (that is supposed to happen soon).

\section{HDACS IN APL: DISTINCT ROLES IN SPACE AND IN TIME}

In contrast to solid tumours, we have a better understanding of the altered function of HDACs in haematological malignancies.

Here, a revisitation of recent results, mainly focusing on acute myeloid leukaemia (AML) models, lead us to additional reasons to explain the complexity of the use of HDAC inhibitors in the clinical setting, linked to distinct effects of HDACs at different stages of tumourigenesis and different action of HDAC inhibitors in distinct tumour cell subtypes.

Acute promyelocytic leukaemia (APL) was one of the first diseases in which the involvement of HDACs was demonstrated mechanistically. Acute promyelocytic leukaemia is characterised by the block of myeloid differentiation at the promyelocytic stage, and is associated with the chromosomal translocation $t(15 ; 17)$, to generate the PML-RAR fusion protein of retinoic acid receptor- $\alpha$ (RAR) with the promyelocytic leukaemia protein (PML). Retinoic acid (RA) showed clinical efficacy in APL patients before the demonstration that it acted by direct targeting of the oncogenic fusion protein (Minucci and Pelicci, 2006).

Subsequent molecular studies placed RA treatment of APL among the best characterised examples of both 'transcription therapy' (whereby the drug targets specifically the oncogenic transcription factor and its aberrant action) and 'differentiation therapy', which reprograms leukaemic cells for terminal differentiation (Tallman et al, 1997; Huang et al, 1988). For all these reasons, although it is a rare disease, APL has been for several years an important model system for learning lessons that can be potentially expanded to other forms of cancer.

In normal cells, RAR acts as a transcription factor, regulating myeloid differentiation and binding in a heterodimeric form with the retinoid $\mathrm{X}$ receptor-specific DNA sequences (called RA-responsive element) found at RAR target genes. In the absence of RA, RAR is found in association with HDAC-containing complexes and represses transcription: RA leads to a conformational switch that causes the release of the corepressor complexes and binding of transcriptional coactivators, with consequent transcription of RAR target genes.

In APL cells, physiological concentrations of RA do not result in the release of HDAC-corepressor complexes from PML-RAR, leading to altered regulation of RAR target genes and of additional PML-RAR-specific targets, and subsequent differentiation block (Minucci and Pelicci, 2006), whereas pharmacological doses of RA (10- to 100 -fold higher than physiological concentrations) reverse the action of the fusion protein, owing to induction of its degradation, and lead to reactivation of the differentiation programme of APL cells. In patients, however, treatment with RA leads only to a transient remission of the disease but cannot entail a definitive cure unless RA is combined to other drugs such as chemotherapy or arsenic.

Studies conducted in transgenic mice have demonstrated that the presence of PML-RAR alone is not sufficient by itself to confer full leukaemic potential to haematopoietic cells, and second hits are necessary for leukaemia development; this thus defines a preleukaemic phase where PML-RAR is acting initially, molecularly distinct from the clonal leukaemia that develops at a later stage.

HDACs in time. The model depicted before suggests a critical role for HDACs in APL, as they are required for the fusion protein to arrest leukaemic differentiation, but new results however have shown that the role of HDACs in APL is more complex than previously thought and demonstrated a dual role for HDACs dependent on the stage of disease progression (so changing 'in time').

Indeed, mice transplanted with haematopoietic progenitors derived from PML-RAR transgenic mice, and carrying knockdown of either HDAC1 or HDAC2, showed a strongly reduced preleukaemic phase, with accelerated leukaemia development 

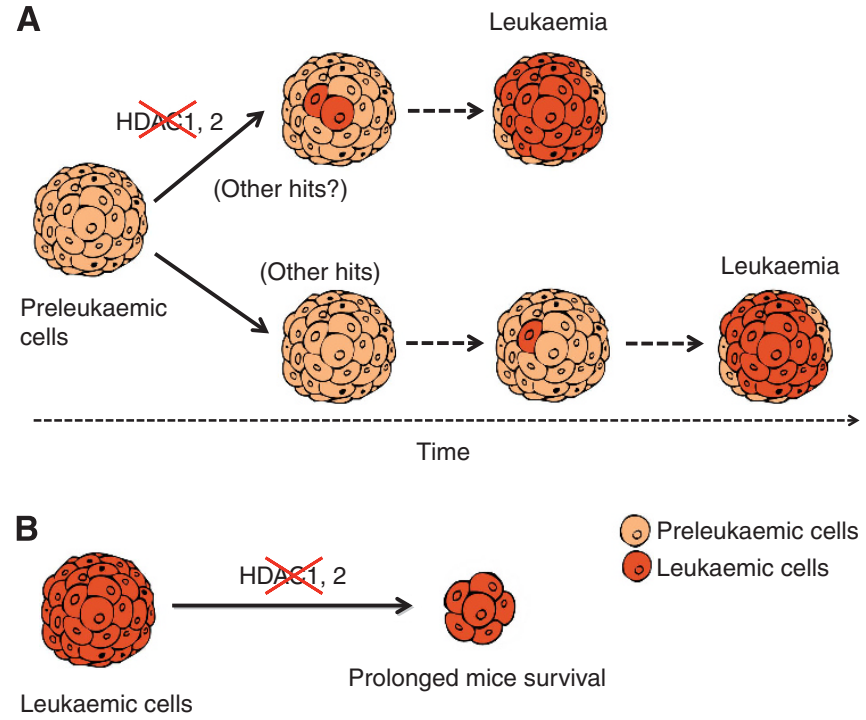

Figure 2. A time-dependent role for HDACs in leukaemia development. (A) During the preleukaemic phase of APL, HDAC1/2 act as tumour suppressors, and their knockdown results in accelerated leukaemia development. This can be because of higher frequency of additional hits, or to direct transformation of PML-RAR preleukaemic cells. (B) During the leukaemic phase, knockdown of HDAC1/2 cause differentiation and then apoptosis of APL cells, with an extended lifespan of the leukaemic mice.

(Figure 2A). Interestingly, HDAC1/2 knockdown caused right after transplantation a marked increase in cells $(G+K+)$ characterised by coexpression of differentiation markers (GR1) and more immature markers (C-kit). This cell sub-population in the absence of HDAC knockdown is found markedly expanded in the leukaemic stage, and is enriched in leukaemia-initiating cells (LICs: see below). Treating mice in the preleukaemic phase with the HDAC inhibitor (HDACi) valproic acid (VPA) mimicked the effect of HDAC1/2 knockdowns.

These results are in striking contrast with those obtained in the leukaemic phase, where knockdown of the same HDACs caused differentiation and apoptosis of APL cells, leading to prolonged mice survival (Figure 2B). Valproic acid treatment induces selectively in leukaemic cell differentiation followed by apoptosis because of the activation of the death receptor pathway. Taken together, these results imply a dual role of HDAC1 and HDAC2 in APL initiation and maintenance, and suggest that they may act as oncosuppressors in the preleukaemic phase, and as oncogenes in leukaemia (Santoro et al, 2013) (Figure 2). Interestingly, HDAC1/HDAC2 knockdown or knockout accelerated development of other tumour types (lymphomas and skin tumours), suggesting that this oncosuppressive role may be more general (Winter et al, 2013).

Not all HDACs share this dual, time-dependent function: HDAC3 acts as an oncogene also during the preleukaemic phase, and its knockdown or inhibition by selective drugs leads to cell differentiation and enhanced apoptosis, and lack of leukaemia development (Matthews et al, 2015).

Several questions remain unanswered: (I) How do HDAC1/ HDAC2 contribute to decrease in the oncogenic potential of PMLRAR-expressing cells in preleukaemia? (II) Is HDAC1/2 loss a second hit sufficient to transform cells, or further hits are required? (III) Most importantly, as HDACs may have an oncosuppressive function, does this imply that clinical treatment with HDACi (such as selective HDAC1i/HDAC2i from those studies discussed above) may favour secondary cancers?

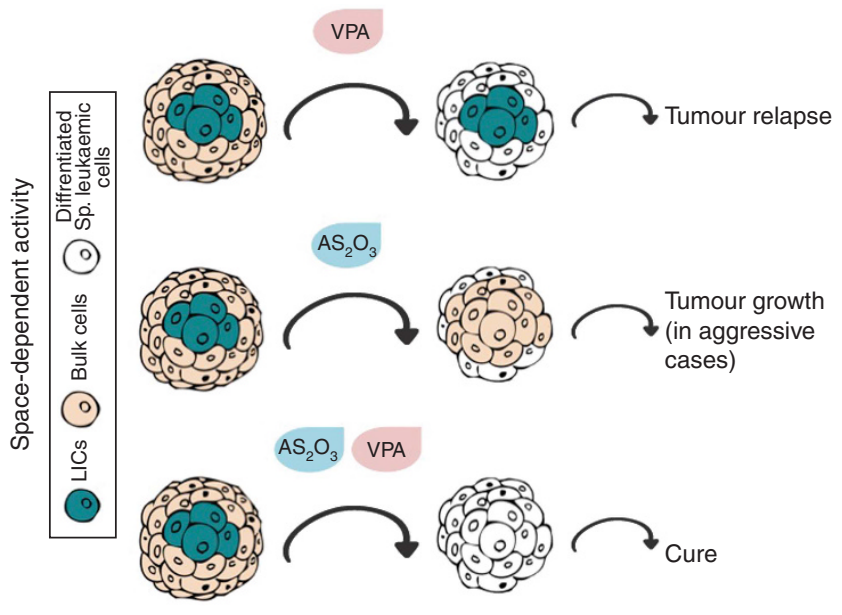

Figure 3. Histone deacetylase inhibitors have distinct effects on tumour cell sub-populations. (Upper panel) Histone deacetylase inhibition by VPA treatment of APL mice results in differentiation of bulk leukaemic cells and prolonged survival, but as LICs are not targeted disease recurs. (Middle panel) Treatment with arsenic leads to tumour regression because of significant reduction in the number of LICs, and progressive tumour exhaustion, but tumour growth in the short term may continue because of bulk of leukaemic cells and - in high-risk aggressive disease forms - lead to patient death. (Bottom panel) Combining the two treatments, by acting on both tumour cell sub-populations, may offer the best perspective in terms of disease control and LIC eradication.

HDACs in space. Most cancers are heterogeneous, and the continuous expansion of the tumour mass is sustained by the self-renewing properties of a sub-population termed 'cancer stem cells' (CSCs) (in leukaemia CSCs are also called LICs) (Kreso and Dick, 2014). The inability of existing therapies (such as chemotherapy) to eradicate CSCs, and to act mainly on the bulk of the tumour mass (that does not proliferate indefinitely) is thought to be one of the most relevant causes for recurrence. Experimental protocols have been set up to measure LICs, based on limiting transplantation experiments in recipient mice of leukaemic cells and recent studies have started to explore the effect of HDACi on different tumour sub-populations in APL.

Valproic acid treatment of APL mice extend their survival, but shortly after interruption of treatment the disease relapses, leading to death; studies to measure the effect of VPA on LICs showed that the number of LICs was not affected by the treatment, consistently with the observed relapse. Valproic acid was therefore selectively acting on the bulk of leukaemic blasts, pushing their differentiation and apoptosis without affecting the self-renewal potential of LICs (Leiva et al, 2012) (Figure 3, upper panel). It remains to be seen if HDACi with different specificities show a different behaviour, and whether genetic experiments (knockdown) will be consistent with the pharmacological observations. We speculate that other HDACs and HDAC-containing complexes not tackled by VPA are essential for LIC maintenance, and therefore HDACs may act differentially in specific tumour cell sub-populations (different tumour 'space').

If we analyse the effect of other drugs on LICs used in APL patients, arsenic treatment was shown to act strongly on LICs (Figure 3, middle panel), and RA showed a dose-dependent phenotype, being more effective on bulk cells at lower doses (Nasr et al, 2008).

It will be of great interest to test in preclinical models the combination of VPA with drugs acting on LICs (such as arsenic trioxide): VPA alone cannot eliminate the disease as it is not targeting LICs, but targeting LICs alone may not necessarily work, as bulk tumour cells can still contribute significantly in the short 
term to tumour growth, leading to patient death before the effect of LIC clearance can be appreciated clinically. Only the combined targeting of the entire tumour cell mass (LICs and bulk) could lead to disease eradication effectively and rapidly, and represents the best potential for cure (Figure 3, bottom panel).

\section{CONCLUSIONS}

Pan-HDACi have given favourable results in a small set of patients with selected haematological diseases, but their use in monotherapy has not been satisfactory. The difference in sensitivity to HDACi cannot be easily allocated to a single cause, making it difficult to envision a smart approach to patient stratification. However, we believe that despite the disappointing results, this field deserves further study and remains a promising therapeutic avenue. Soon, we will know whether more selective HDACi will be more effective in the clinics, and with reduced side effects. The studies in murine models of leukaemia suggest that it is necessary to consider not only the differences among different classes of HDACs but also how the same molecules may act in 'time' and 'space', as we have previously illustrated. In particular, we propose that a systematic effort should be performed to study the effects of HDACi and other epidrugs on the stem cell compartment $v s$ the rest of tumour cells, to devise treatment schemes that combine more efficiently drugs targeting the different tumour cell subpopulations. Of course, this must not be limited to epidrugs, and combination with other agents such as DNA-damaging chemotherapeutic drugs, or proteasome inhibitors, has already shown promising results that could be reinterpreted based on the studies proposed above. Histone deacetylase inhibitors may well find their optimal 'clinical space' in the end.

\section{ACKNOWLEDGEMENTS}

We thank Giorgio Melloni for the essential help in the mutational analysis of HDACs, and Marina Mapelli for suggestions. Work in SM's laboratory is supported by AIRC (Italian Association for Cancer Research), FIRC (Italian Foundation for Cancer Research), Epigen Flagship Project, European Community (4D Cell Fate and Blueprint) and Fondazione Veronesi. For lack of space, it was not possible to mention all of the original papers. A more complete list of references can be found in the following reviews: Minucci and Pelicci (2006), Botrugno et al (2009), Mercurio et al (2010), Falkenberg and Johnstone (2014) and West and Johnstone (2014).

\section{CONFLICT OF INTEREST}

The authors declare no conflict of interest.

\section{REFERENCES}

Botrugno OA, Santoro F, Minucci S (2009) Histone deacetylase inhibitors as a new weapon in the arsenal of differentiation therapies of cancer. Cancer Lett 280(2): 134-144.

Cerami E, Gao J, Dogrusoz U, Gross BE, Sumer SO, Aksoy BA, Jacobsen A, Byrne CJ, Heuer ML, Larsson E, Antipin Y, Reva B, Goldberg AP, Sander C, Schultz N (2012) The cBio cancer genomics portal: an open platform for exploring multidimensional cancer genomics data. Cancer Discovery 2(5): 401-404.

Chiaradonna F, Cirulli C, Palorini R, Votta G, Alberghina L (2015) New insights into the connection between histone deacetylases, cell metabolism, and cancer. Antioxid Redox signal 23(1): 30-50.
Dawson MA, Kouzarides T (2012) Cancer epigenetics: from mechanism to therapy. Cell 150(1): 12-27.

Eot-Houllier G, Fulcrand G, Magnaghi-Jaulin L, Jaulin C (2009) Histone deacetylase inhibitors and genomic instability. Cancer Lett 274(2): 169-176.

Falkenberg KJ, Johnstone RW (2014) Histone deacetylases and their inhibitors in cancer, neurological diseases and immune disorders. Nat Rev Drug Discov 13(9): 673-691.

Fraga MF, Ballestar E, Villar-Garea A, Boix-Chornet M, Espada J, Schotta G, Bonaldi T, Haydon C, Ropero S, Petrie K, Iyer NG, Perez-Rosado A, Calvo E, Lopez JA, Cano A, Calasanz MJ, Colomer D, Piris MA, Ahn N, Imhof A, Caldas C, Jenuwein T, Esteller M (2005) Loss of acetylation at Lys 16 and trimethylation at Lys20 of histone $\mathrm{H} 4$ is a common hallmark of human cancer. Nat Genet 37(4): 391-400.

Haberland M, Montgomery RL, Olson EN (2009) The many roles of histone deacetylases in development and physiology: implications for disease and therapy. Nat Rev Genet 10(1): 32-42.

Huang ME, Ye YC, Chen SR, Chai JR, Lu JX, Zhoa L, Gu LJ, Wang ZY (1988) Use of all-trans retinoic acid in the treatment of acute promyelocytic leukemia. Blood 72: 567-572.

Insinga A, Monestiroli S, Ronzoni S, Carbone R, Pearson M, Pruneri G, Viale G, Appella E, Pelicci P, Minucci S (2004) Impairment of p53 acetylation, stability and function by an oncogenic transcription factor. EMBO J 23(5): 1144-1154.

Kebede AF, Schneider R, Daujat S (2015) Novel types and sites of histone modifications emerge as players in the transcriptional regulation contest. FEBS J 282(9): 1658-1674.

Kreso A, Dick JE (2014) Evolution of the cancer stem cell model. Cell Stem Cell 14(3): 275-291.

Leiva M, Moretti S, Soilihi H, Pallavicini I, Peres L, Mercurio C, Dal Zuffo R, Minucci S, de The H (2012) Valproic acid induces differentiation and transient tumor regression, but spares leukemia-initiating activity in mouse models of APL. Leukemia 26(7): 1630-1637.

Matthews GM, Mehdipour P, Cluse LA, Falkenberg KJ, Wang E, Roth M, Santoro F, Vidacs E, Stanley K, House CM, Rusche JR, Vakoc CR, Zuber J, Minucci S, Johnstone RW (2015) Functional-genetic dissection of HDAC dependencies in mouse lymphoid and myeloid malignancies. Blood 126(21): 2392-2403.

Mercurio C, Minucci S, Pelicci PG (2010) Histone deacetylases and epigenetic therapies of hematological malignancies. Pharmacol Res 62(1): $18-34$.

Minucci S, Pelicci PG (2006) Histone deacetylase inhibitors and the promise of epigenetic (and more) treatments for cancer. Nat Rev Cancer 6(1): 38-51.

Müller BM, Jana L, Kasajima A, Lehmann A, Prinzler J, Budczies J, Winzer KJ, Dietel M, Weichert W, Denkert C (2013) Differential expression of histone deacetylases HDAC1, 2 and 3 in human breast cancer - overexpression of HDAC2 and HDAC3 is associated with clinicopathological indicators of disease progression. BMC Cancer 13: 215.

Nasr R, Guillemin MC, Ferhi O, Soilihi H, Peres L, Berthier C, Rousselot P, Robledo-Sarmiento M, Lallemand-Breitenbach V, Gourmel B, Vitoux D, Pandolfi PP, Rochette-Egly C, Zhu J, de Thé H (2008) Eradication of acute promyelocytic leukemia-initiating cells through PML-RARA degradation. Nat Med 14(12): 1333-1342.

Rincon-Arano H, Halow J, Delrow JJ, Parkhurst SM, Groudine M (2012) UpSET recruits HDAC complexes and restricts chromatin accessibility and acetylation at promoter regions. Cell 161(6): 1214-1228.

Ropero S, Fraga MF, Ballestar E, Hamelin R, Yamamoto H, Boix-Chornet M, Caballero R, Alaminos M, Setien F, Paz MF, Herranz M, Palacios J, Arango D, Orntoft TF, Aaltonen LA, Schwartz Jr S, Esteller M (2006) A truncating mutation of HDAC2 in human cancers confers resistance to histone deacetylase inhibition. Nat Genet 38(5): 566-569.

Saji S, Kawakami M, Hayashi S, Yoshida N, Hirose M, Horiguchi S, Itoh A, Funata N, Schreiber SL, Yoshida M, Toi M (2005) Significance of HDAC6 regulation via estrogen signaling for cell motility and prognosis in estrogen receptor-positive breast cancer. Oncogene 24(28): 4531-4539.

Santoro F, Botrugno OA, Dal Zuffo R, Pallavicini I, Matthews GM, Cluse L, Barozzi I, Senese S, Fornasari L, Moretti S, Altucci L, Pelicci PG, Chiocca S, Johnstone RW, Minucci S (2013) A dual role for Hdac1: oncosuppressor in tumorigenesis, oncogene in tumor maintenance. Blood 121(17): 3459-3468. 
Subramanian S, Bates S, Wright J, Espinoza-Delgado I, Piekarz R (2010) Clinical toxicities of histone deacetylase inhibitors. Pharmaceuticals 3 : 2751-2767.

Tallman MS, Andersen JW, Schiffer CA, Appelbaum FR, Feusner HJ, Ogden A, Schepherd L, Willman C, Bloomfield CD, Rowe JM, Wiernik PH (1997) All-trans-retinoic acid in acute promyelocytic leukemia. N Engl J Med 337(15): 1021-1028.

Valente S, Mai A (2014) Small-molecule inhibitors of histone deacetylase for the treatment of cancer and non-cancer disease: a patent review (2011-2013). Expert Opin Therap Patents 24(4): 401-415.

Wagner GR, Payne RM (2013) Widespread and enzyme-independent $N \varepsilon$-acetylation and $N \varepsilon$-succinylation of proteins in the chemical conditions of the mitochondrial matrix. J Biol Chem 288(40): 29036-29045.

Wang Z, Zang C, Cui K, Schones DE, Barski A, Peng W, Zhao K (2009) Genome-wide mapping of HATs and HDACs reveals distinct functions in active and inactive genes. Cell 138(5): 1019-1031.
West AC, Johnstone RW (2014) New and emerging HDAC inhibitors for cancer treatment. J Clin Invest 124(1): 30-39.

Winter M, Moser MA, Meunier D, Fischer C, Machat G, Mattes K, Lichtenberger BM, Brunmeir R, Weissmann S, Murko C, Humer C, Meischel T, Brosch G, Matthias P, Sibilia M, Seiser C (2013) Divergent roles of HDAC1 and HDAC2 in the regulation of epidermal development and tumorigenesis. EMBO J 32(24): 3176-3191.

Yang XJ, Seto E (2008) The Rpd3/Hda1 family of lysine deacetylases: from bacteria and yeast to mice and man. Nat Rev Mol Cell Biol 9(3): 206-218.

(c) (1)(2) This work is licensed under the Creative Commons (c) 1 BY NG SA Attribution-Non-Commercial-Share Alike 4.0 International License. To view a copy of this license, visit http:// creativecommons.org/licenses/by-nc-sa/4.0/

Supplementary Information accompanies this paper on British Journal of Cancer website (http://www.nature.com/bjc) 